\title{
ON THE RELATIONS BETWEEN TAUT, TIGHT AND HYPERBOLIC MANIFOLDS
}

\author{
BY PETER KIERNAN
}

\author{
Communicated by Richard Palais, May 22, 1969
}

In [1], Professor Kobayashi defined hyperbolic and complete hyperbolic manifolds. In [2], Professor Wu defined tight and taut complex manifolds. The purpose of this paper is to show that these concepts are related in the following way:

$$
\begin{aligned}
& \text { complete hyperbolic } \Rightarrow \text { taut } \\
& \text { taut } \underset{\not}{\Rightarrow} \text { hyperbolic } \\
& \text { hyperbolic } \Leftrightarrow \text { tight (with respect to some metric) }
\end{aligned}
$$

It seems likely that taut implies complete hyperbolic, but I cannot prove that at the present time. Don Eisenman has obtained these results concurrently by a slightly different method.

We begin by recalling the definition of the Kobayashi pseudodistance $d_{M}$ associated to the complex manifold $M$. Let $p$ and $q$ be points in $M$. By a chain $\alpha$ from $p$ to $q$, we mean a sequence $p=p_{0}, p_{1}$, $\cdots, p_{k}=q$ of points in $M$, points $a_{1}, \cdots, a_{k}$ in the unit disk $D$ $=\{z \in C|| z \mid<1\}$ and holomorphic maps $f_{1}, \cdots, f_{k}$ of $D$ into $M$ with $f_{i}(0)=p_{i-1}$ and $f_{i}\left(a_{i}\right)=p_{i}$. The length $|\alpha|$ of $\alpha$ is defined by

$$
|\alpha|=\sum_{i=1}^{k} d\left(0, a_{i}\right)=\sum_{i=1}^{k} \log \frac{1+\left|a_{i}\right|}{1-\left|a_{i}\right|}
$$

where $d$ is the Poincaré-Bergman distance on $D$. It is given by the metric $d s^{2}=d z d \bar{z} /\left(1-|z|^{2}\right)^{2}$. We set $d_{M}(p, q)=\inf _{\alpha \in A}|\alpha|$, where $A$ is the set of all chains from $p$ to $q$. It is easy to see that $d_{M}$ is a pseudodistance on $M$. If $d_{M}$ is an actual distance, we say that $M$ is hyperbolic. $M$ is called complete hyperbolic if $d_{M}$ is a complete metric, i.e., if all Cauchy sequences converge. Kobayashi (see $[1, \S 8]$ ) has shown that complete hyperbolic implies that all bounded subsets have compact closure.

It follows immediately from the definition of $d_{M}$ and $d_{N}$, that if $f: M \rightarrow N$ is holomorphic and $p, q \in M$, then $d_{N}(f(p), f(q)) \leqq d_{M}(p, q)$. The classical Schwarz-Pick lemma implies that the Kobayashi distance $d_{D}$ on the unit disk $D$ is the same as the Poincaré-Bergman distance $d$. 
Let $\mathbb{Q}(N, M)$ denote the set of holomorphic maps from $N$ into $M$. A sequence $\left\{f_{i}\right\}$ in $a(N, M)$ is called compactly divergent if given any compact $K$ in $N$ and compact $K^{\prime}$ in $M$, there exists $j$ such that $f_{i}(K)$ $\bigcap K^{\prime}=\varnothing$ for all $i \geqq j$. Fix a metric $\rho$ on $M$ which induces its topology. $Q(N, M)$ is called normal if every sequence in $Q(N, M)$ contain a subsequence which is either uniformly convergent on compact sets or compactly divergent. $M$ is said to be taut if $a(N, M)$ is normal for every $N .(M, \rho)$ is said to be tight if $a(N, M)$ is equicontinuous for every $N$. It should be noted that tautness is an intrinsic property of $M$, while tightness depends on the metric $\rho$.

For the rest of the paper, we shall use the following notation.

(1) $p$ and $q$ are distinct points of $M$.

(2) $B=\left\{\left.\left(w_{1}, \cdots, w_{n}\right)|| w_{1}\right|^{2}+\cdots+\left|w_{n}\right|^{2}<1\right\}$ is a coordinate neighborhood centered at $p$, such that $q \notin B$.

(3) $B_{s}=\left\{\left.\left(w_{1}, \cdots, w_{n}\right)|| w_{1}\right|^{2}+\cdots+\left|w_{n}\right|^{2} \leqq s^{2}<1\right\} \subset B$.

(4) $\rho$ is a metric on $M$ which induces its topology.

(5) $V_{s}=\left\{p^{\prime} \in M \mid \rho\left(p^{\prime}, p\right)<s\right\}$.

(6) $D_{\delta}=\{z \in D|| z \mid<\delta<1\}$.

An ordered pair $(r, \delta)$ of strictly positive numbers is said to satisfy property $A$ (relative to the choices above) if for every holomorphic map $f: D \rightarrow M$ with $f(0) \in B_{r}$, we have $f\left(D_{\delta}\right) \subset B$.

LemмA. Let $M, p, q$ and $B$ be as above. If there exists a pair $(r, \delta)$ satisfying property $A$, then $d_{M}(p, q)>0$.

Proof. Choose a constant $c>0$ such that $d_{D}(0, a) \geqq c d_{D_{\delta}}(0, a)$ for all $a \in D_{\delta / 2}$.

Let $\alpha=\left\{p=p_{0}, p_{1}, \cdots, p_{l}=q ; a_{1}, \cdots, a_{l} ; f_{1}, \cdots, f_{l}\right\}$ be a chain from $p$ to $q$. Without loss of generality, we can assume that $a_{1}, \cdots$, $a_{k} \in D_{\delta / 2}, p_{0}, p_{1}, \cdots, p_{k} \in B_{r}$ and that $p_{k} \in \partial B_{r}$. Now

$$
\begin{aligned}
|\alpha| & \geqq \sum_{i=1}^{k} d_{D}\left(0, a_{i}\right) \geqq c \sum_{i=1}^{k} d_{D_{\delta}}\left(0, a_{i}\right) \geqq c \sum_{i=1}^{k} d_{B}\left(p_{i-1}, p_{i}\right) \\
& \geqq c d_{B}\left(0, p_{K}\right)=c^{\prime} \quad \text { where } c^{\prime} \text { is constant }>0 .
\end{aligned}
$$

Thus $d_{M}(p, q) \geqq c^{\prime}>0$. Q.E.D.

Proposition 1. If $(M, \rho)$ is tight, then $M$ is hyperbolic. If $M$ is hyperbolic, then $\left(M, d_{M}\right)$ is tight.

Proof. Assume $(M, \rho)$ is tight. Using the notation above, there exists $\epsilon>0$ such that $V_{2 \epsilon} \subset B$. Since $Q(D, M)$ is equicontinuous, there exists $\delta>0$ such that if $f: D \rightarrow M$ is holomorphic with $f(0) \in V_{\epsilon}$, then $f\left(D_{\delta}\right) \subset V_{2 \epsilon} \subset B$. Choose $r>0$ such that $B_{r} \subset V_{\epsilon}$. Then $(r, \delta)$ satisfies 
property $A$. By the lemma, $d_{M}(p, q)>0$. Since $p$ and $q$ were arbitrary distinct points, $M$ is hyperbolic. The second statement is trivial. Q.E.D.

Proposition 2. If $M$ is taut, then $M$ is hyperbolic.

Proof. Assume $M$ is not hyperbolic. Then there exist distinct points $p$ and $q$ with $d_{M}(p, q)=0$. By the lemma, $\left(\frac{1}{2}, 1 / n\right)$ does not satisfy property $A$ for any $n$. Thus there exists a holomorphic map $f_{n}: D \rightarrow M$ with $f(0) \in B_{1 / 2}$ and $f_{n}\left(D_{1 / n}\right) \nsubseteq B$. The sequence $\left\{f_{i}\right\}$ has no subsequence which is either uniformly convergent on compact sets or compactly divergent. Thus $M$ is not taut. Q.E.D.

EXAMPLE. $D \times D-\{(0,0)\}$ is hyperbolic, but it is neither taut nor complete hyperbolic.

Proposition 3. If $M$ is complete hyperbolic, then $M$ is taut.

Proof. Let $N$ be another manifold. Since $\left(M, d_{M}\right)$ is tight, $a(N, M)$ is equicontinuous. Since $M$ is complete hyperbolic, every bounded set in $M$ is relatively compact. This implies that $Q(N, M)$ is normal (see Lemma 1.1 in [2]). Thus $M$ is taut. Q.E.D.

Finally, we observe that if $M$ is a hyperbolic Riemann surface, then $M$ is complete hyperbolic. This follows from the fact that $M$ is covered by $D$ which is complete hyperbolic. By Proposition 5.5 of [1], $M$ is complete hyperbolic.

\section{REFERENCES}

1. S. Kobayashi, Invariant distances on complex manifolds and holomorphic maps, J. Math. Soc. Japan 19 (1967), 460-480. MR 38 \#736.

2. H. Wu, Normal families of holomorphic mappings, Acta Math. 119 (1967), 193233. MR 37 \#468.

University of California, Berkeley, California 94720 